\title{
$A b$ Initio Study of 17a-Ethinylestradiol and Estrone Molecules Interacting with Single Wall Carbon Nanotube
}

\author{
Shuanne M. Castro, ${ }^{a}$ Herisson C. Ribeiro, ${ }^{a}$ Adriana B. Araújo ${ }^{b}$ and Silvete Guerini ${ }^{\circledR *, a}$ \\ ${ }^{a}$ Departamento de Física, Universidade Federal do Maranhão, 65080-805 São Luís-MA, Brazil \\ ${ }^{b}$ Instituto Federal do Maranhão, Campus São Luís, Centro Histórico, 65010-500 São Luís-MA, Brazil
}

\begin{abstract}
In this work, a systematic study of $17 \alpha$-ethinylestradiol and estrone molecules interacting with single wall carbon nanotube is presented by means of first principle calculations. The structural properties, binding energy, charge transfer, and electronic properties were calculated and analyzed to characterize the effect of interaction of $17 \alpha$-ethinylestradiol and estrone molecules on the nanotube surface. Results show that the molecular structure and electronic character of single wall carbon nanotube were not affected due to the presence of the molecules. It is observed via binding energy calculated that ethinylestradiol and estrone molecules interact weakly with the nanotube. The results are very interesting once that they suggest that nanotubes are sensitive to $17 \alpha$-ethinylestradiol and estrone molecules which can lead to confection of sensor to detect these molecules.
\end{abstract}

Keywords: nanotube, ethinylestradiol, estrone, DFT

\section{Introduction}

Due to advancement of technology and knowledge about the effects of pollution in water environments, some countries have sought to expand regulation and monitoring of endocrine disrupter. Personal care and pharmaceuticals products have been recognized as contaminants of emerging concern due to their frequent presence in aquatic environments. ${ }^{1}$ These products are known to be released into aquatic environments through domestic wastewater, hospital discharges, improper manufacturer disposal, and others. Among contaminants of water, the hormones stand out once their use has increasing. The hormones are used as a form of hormonal treatment for problems related to menopause, polycystic ovarian syndrome, among other forms. The human beings excrete a considered quantity of hormones every day, in addition to which some hormones are used in the industry for example to accelerate the growth of animals. ${ }^{2}$ It is referred to as endocrine disruptors, as products that interfere with the reproductive and hormonal systems of animals and humans, among which we can highlight $17 \alpha$-ethinylestradiol and estrone. Exposure of humans and animals to substances of the environment with estrogenic action may result in adverse changes

*e-mail: silvete.guerini@gmail.com in reproductive, functional or behavioral development. There is research suggesting that increased incidence of breast cancer, decreased sperm count, decreased fertility, congenital defects to fetal and other changes are associated with exposure to environmental chemicals with estrogen action. Studies show the presence of the estrogenic compound in fish feminization and hermaphroditism. The estrone compound is a synthetic hormone derived from estradiol. The presence of the ethinyl group makes it to have a greater resistance to biodegradation. ${ }^{3-7}$

Removal of this type of water contaminants is a complicated process because depends on the chemical and biological properties of pollutants. ${ }^{8}$ Adsorption has been prevalent and is often used to the removal of pollutants in water considering different adsorbents. Carbon nanotubes (CNTs) have great benefits in several fields, such as in the industry, ${ }^{9}$ for the treatment of diseases and transport of medicines, salinization of water, gas sensors ${ }^{10-12}$ and medical applications. ${ }^{13-15} \mathrm{CNTs}$ are showing great potential for the removal or detection of toxic molecules, whether in aqueous or gaseous medium. Their properties as large surface areas, high chemical and thermal stability make these nanomaterials attractive to decontaminate water and wastewater. ${ }^{16}$

Teixeira et al., ${ }^{17}$ in an experimental work, demonstrated that hybrids composites based on carbon nanotubes and 
nanofibers supported on $\mathrm{Mg}$ silicate fibers derived from chrysotile are highly efficient adsorbent of the ethinylestradiol. The efficiency is due to the highly hydrophobic surface of the carbon nanotube and nanofibers completely exposed and available for the adsorption of ethinylestradiol and also the amphiphilic character of the composite which allows a facile transport of the ethinylestradiol from the aqueous media. On the other hand, the adsorbents are magnetic and can be easily removed from the aqueous medium through a magnetic separation process. ${ }^{17}$

Studies performed through adsorption experiment and theoretical calculations show multi-walled and single-walled carbon nanotube are systems successful as nanoadsorbents for removal of some dye from aqueous solutions. ${ }^{18,19}$ The adsorption procedure is an efficient method for the removal of synthetic dyes from effluents once that this process transfers dyes from the water effluent to a solid phase notably decreasing dye bioavailability to live organisms. Jing et al. ${ }^{20}$ used the molecular simulation to investigate the influence of polarity of polymer chains, i.e., the effect of polarity of molecules on filling polymer chains into single wall carbon nanotubes (SWCNTs). The simulations show that polarity of molecules have an important role in the filling phenomena.

In $a$ priori work, ${ }^{21}$ the authors investigate the effects on the electronic properties of SWCNTs interacting with triclosan molecule using first-principle calculation in different configurations. The results show that carbon nanotubes are promising for triclosan molecule adsorption since it is sensitive to the presence of triclosan molecule and could be used as a filter for wastewater treatment.

Aiming to understand the adsorption mechanism of $17 \alpha$-ethinylestradiol and estrone molecules on an SWCNTs surface, we studied the interaction between the $17 \alpha$-ethinylestradiol and estrone molecules and SWCNTs using first principle calculations based on density functional theory (DFT). Our calculations predict that $17 \alpha$-ethinylestradiol and estrone interacts with SWCNTs through the physical process in all considered configurations.

\section{Methodology}

The structural, energetic and electronic properties of $17 \alpha$-ethinylestradiol and estrone molecules interacting with a pristine (8.0) SWCNT, in different configurations, were simulated using first principle calculations based on DFT $^{22}$ and implemented in Siesta code. ${ }^{23}$ The Siesta code ${ }^{23}$ uses a linear combination of pseudoatomic orbitals with numerical atomic basis set to solve the Kohn-Sham equations. ${ }^{24}$ Double zeta basis set with polarization function was used in all calculations. For exchange and correlation terms, local density approximation as parameterization of Perdew and Zunger $^{25}$ was used. The interaction between ionic cores and valence electrons was described by norm conserving pseudopotentials ${ }^{26}$ in Kleinamn-Bylander form. ${ }^{27}$ A cutoff of 150 Ry for the grid integration was adopted to represent the charge density. The electronic relaxation was converged to $1.0 \times 10^{-4}$. Twenty three Monkhorst-Pack k-points were employed for the Brillouin zone integration, along the $\Gamma-\mathrm{X}$ directions. ${ }^{28}$

We employed one-dimensional periodic boundary conditions along the nanotube axis. The pristine (8.0) SWCNTs with 192 carbon atoms correspond to a length of $24.16 \AA$ in the supercell. The lateral separation was of $20.00 \AA$ to ensure that the tube and the molecules did not interact with their periodic images. The positions of all atoms were optimized using a conjugated gradient algorithm until the residual force acting on each atom was less than $0.04 \mathrm{eV}^{-1}$.

The binding energies $\left(E_{b}\right)$ between nanotubes and molecules were calculated using the basis set superposition error (BSSE). ${ }^{29}$ This correction is done through the counterpoise method using "ghost" atoms, as the following equation:

$$
\begin{aligned}
& \mathrm{E}_{\mathrm{b}}=\mathrm{E}_{\mathrm{T}}[\mathrm{SWCNT}+\mathrm{M}]-\mathrm{E}_{\mathrm{T}}[\mathrm{SWCNT}+\text { Mghost }]- \\
& \mathrm{E}_{\mathrm{T}}[\text { SWCNTghost }+\mathrm{M}]
\end{aligned}
$$

where $\mathrm{E}_{\mathrm{T}}[\mathrm{SWCNT}+\mathrm{M}]$ is the total energy of SWCNT plus (17 $\alpha$-ethinylestradiol or estrone) molecules. The "ghost" species refers to additional basis wave functions centered at the position of the molecules $\mathrm{M}$ or the nanotube, but without any atomic potential. The values for the minimum distances (d) between the molecules and nanotube were obtained from a plane tangent to the SWCNT to the nearest atom of the molecules.

\section{Results and Discussion}

The geometry of (8.0) SWCNT plus $17 \alpha$-ethinylestradiol (estrone) molecule, in different configurations, are optimized to find the most stable adsorption configuration. In both systems we optimized thirteen configurations to find the most stable configuration. In Figure 1 it is shown the four most stable configurations of SWCNT interacting with $17 \alpha$-ethinylestradiol molecule. The configurations considered are $17 \alpha$-ethinylestradiol molecule parallel to axis of nanotube (Figure 1a), the 17 $\alpha$-ethinylestradiol molecule perpendicular to the axis of the nanotube with the $\mathrm{H}$ atoms close to the tube (Figure 1b), the $17 \alpha$-ethinylestradiol molecule perpendicular to the axis of 

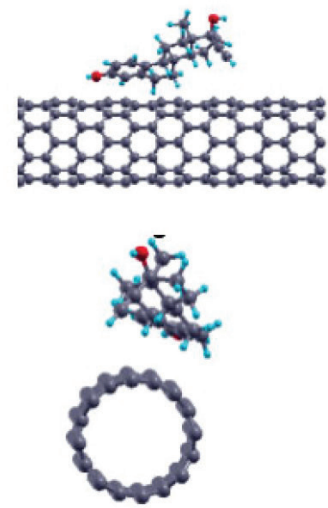

(a)
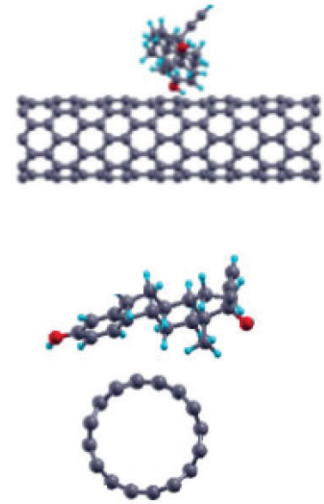

(b)

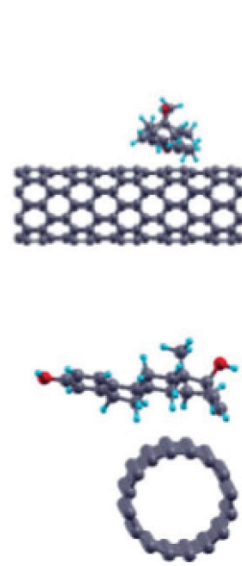

(c)

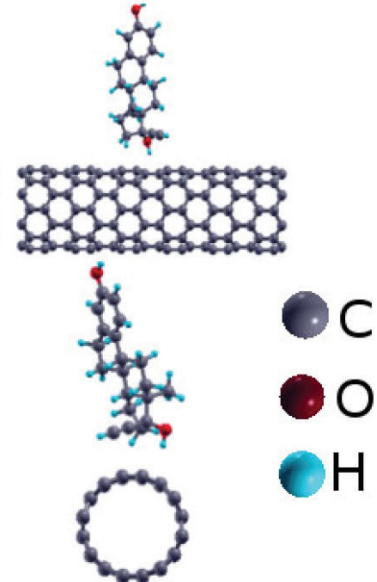

(d)

Figure 1. Schematic view of four most stable configurations considered for 17 $\alpha$-ethinylestradiol interaction with (8.0) SWCNT to references.

the tube with the ethinyl group close to the tube (Figure 1c) and the $17 \alpha$-ethinylestradiol molecule with atoms of the $\mathrm{H}, \mathrm{C}$ and $\mathrm{O}$ close to nanotube (Figure 1d).

Table 1 summarizes the calculated binding energies $\left(E_{b}\right)$ according to equation 1, binding distance (d), energy gap $\left(\mathrm{E}_{\mathrm{g}}\right)$ and charge transfer $(\mathrm{CT})$ for all the studied configurations shown in Figure 1. The values for d between the $17 \alpha$-ethinylestradiol molecule and SWCNT were obtained from plane tangent to the nanotube to the nearest atom of the molecule. Negative values from $\mathrm{E}_{\mathrm{b}}$ and $\mathrm{CT}$ indicate an attraction between the SWCNT and the molecule, and a charge transfer from SWCNT to molecule, respectively.

Table 1. Binding energies $\left(E_{b}\right)$, distance of binding (d), gap energy $\left(E_{g}\right)$, and charge transfer (CT) calculated for different adsorption sites of (8.0) SWCNT, as shown in Figure 1. The minus sign in the charge transfer values indicates that $17 \alpha$-ethinylestradiol molecule receives electronic charge

\begin{tabular}{lcccc}
\hline System & $\mathrm{E}_{\mathrm{b}} / \mathrm{eV}$ & $\mathrm{d} / \AA$ & $\mathrm{E}_{\mathrm{g}} / \mathrm{eV}$ & $\mathrm{CT} / \mathrm{e}^{-}$ \\
\hline $\mathrm{a}$ & -0.45 & 2.39 & 0.58 & -0.11 \\
$\mathrm{~b}$ & -0.43 & 2.54 & 0.57 & -0.13 \\
$\mathrm{c}$ & -0.40 & 2.71 & 0.59 & -0.17 \\
$\mathrm{~d}$ & -0.35 & 2.35 & 0.58 & -0.16 \\
\hline
\end{tabular}

Thus, according to Table 1, all studied configurations exhibited, in average, $\mathrm{E}_{\mathrm{b}}$ around $0.40 \mathrm{eV}$ (in modulus), which corresponds to a physical interaction. The analysis shows that the most stable configuration was the one in which the $17 \alpha$-ethinylestradiol molecule is localized parallel to axis of nanotube (Figure 1a). The negative values for binding energy indicate a force of attraction between the 17 $\alpha$-ethinylestradiol molecule and SWCNT.

From the bands energy of Figure 2P, it can be observed that pristine (8.0) SWCNT behaved like a semiconductor system, displaying a small difference of $0.60 \mathrm{eV}$ between the valence and conduction bands. These results are in complete agreement with the previous determinations reported in literature. ${ }^{21}$ Figures $2 \mathrm{a}-2 \mathrm{e}$ show the bands energy of all configurations shown in Figures 1a-1e. It was observed that the electronic band of pristine SWCNT change little with interaction of $17 \alpha$-ethinylestradiol molecule. One significant change observed was that there were mixtures between the levels of $17 \alpha$-ethinylestradiol molecule and nanotube mainly in valence band.

Figure 2a shows the band structure for the most stable configuration (Figure 1a), from which structures it can be observed that partially delocalized levels appear in approximately $-0.81 \mathrm{eV}$ below the Fermi level due to an interaction between $17 \alpha$-ethinylestradiol molecule and (8.0) SWCNT. In band structure of Figure 2b, which refers to the configuration of Figure 1b, partially delocalized level also appears in $0.70 \mathrm{eV}$ below Fermi level. However, bands structure of Figures $2 \mathrm{c}$ and $2 \mathrm{~d}$ presented localized level in $0.58 \mathrm{eV}$ below the Fermi level, that correspond to the configurations of Figures 1c and 1d. The systems remaining semiconductor in studied configurations with energy gap close to pristine SWCNT (see Table 1).

Figures 3a-3d show the local density of states (LDOS) plot of $17 \alpha$-ethinylestradiol molecule adsorbed on the (8.0) SWCNT for all configurations (shown in Figure 1) presented for the electronic levels around the Fermi level (see Figures 2a-2d). From these LDOS plots, we observed that the largest contribution in this region belongs to $17 \alpha$-ethinylestradiol molecule, showing a weak interaction between $17 \alpha$-ethinylestradiol molecule and (8.0) SWCNT.

In order to predict the interaction between the estrone molecule and (8.0) SWCNT in different configurations (Figure 4) we calculated the binding energy between molecule and nanotube using the equation 1 . It was found that estrone molecule prefers to be attached on surface 

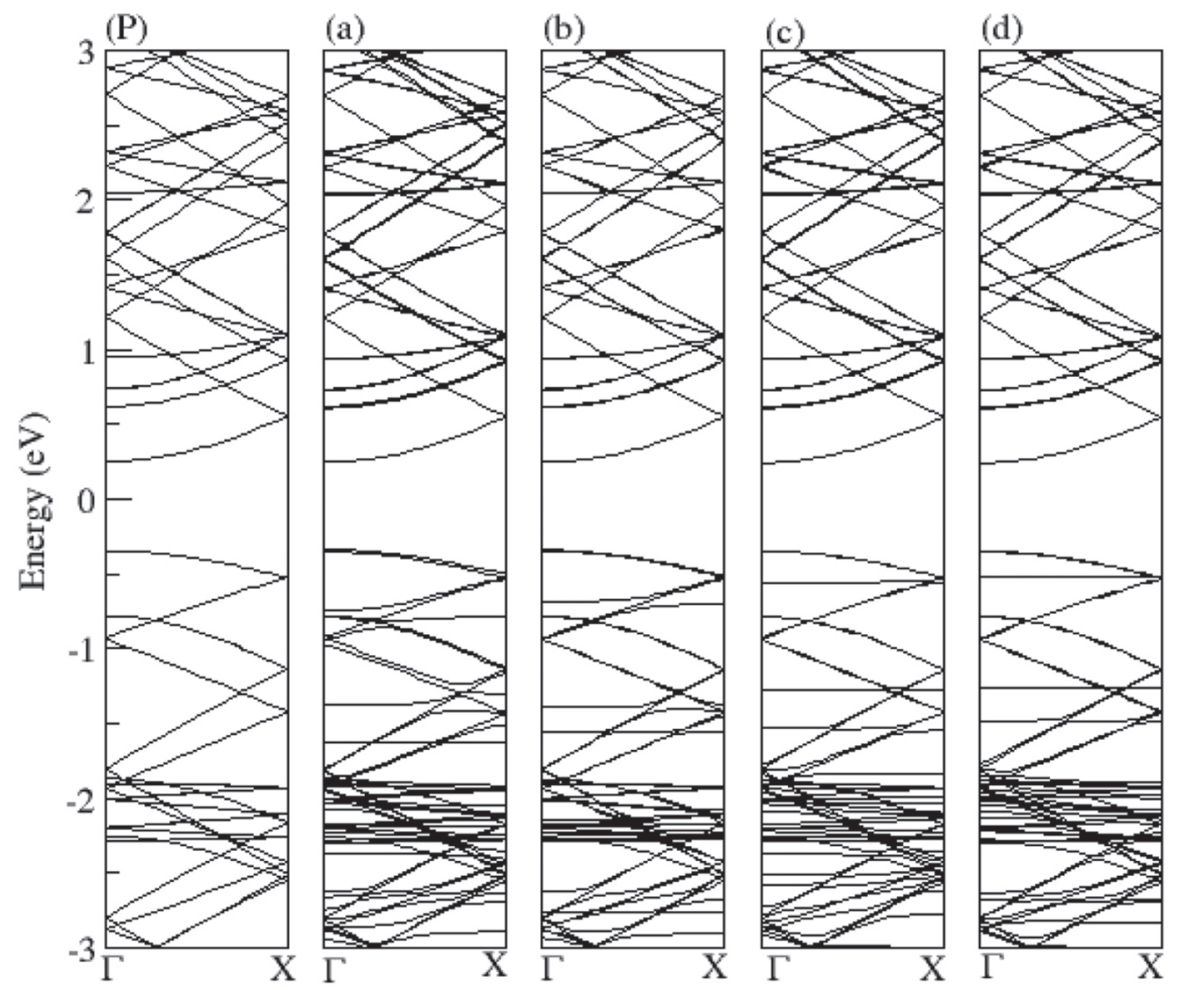

Figure 2. Electronic band structure for (P) pristine (8.0) SWCNT and (a-d) for 17 $\alpha$-ethinylestradiol molecule adsorbed on (8.0) SWCNT in configurations shown in Figures 1a-1d, respectively. The Fermi level is localized in zero.
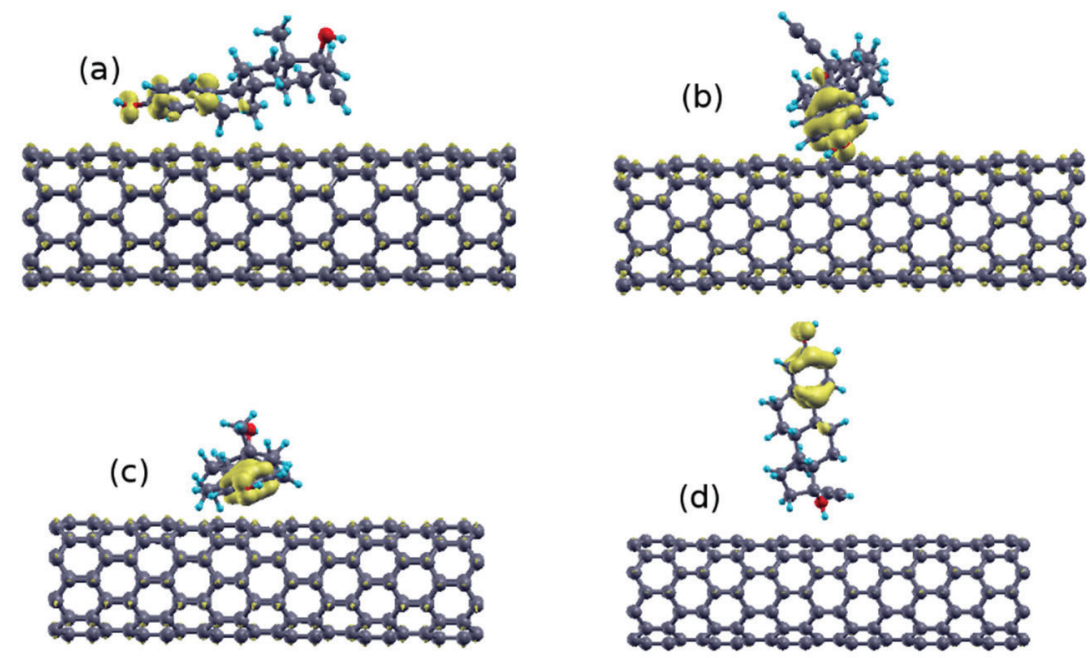

Figure 3. (a-d) Plot of the local density of states, around the Fermi level, for (8.0) SWCNT interacting with 17 $\alpha$-ethinylestradiol molecule for the electronic configurations shown in Figures 2a-2d, respectively. An isosurface $0.005 \mathrm{e}^{-1}$ bohr $^{-3}$ was considered.

of the tube with parallel orientation with respect to the nanotube axis (see Figure 4a). After adsorption of molecule, the interaction distance between the closest atoms of the estrone molecule and SWCNT is around $2.49 \AA$ and the $\mathrm{C}-\mathrm{C}$ bond length near the adsorbate is not changed. The corresponding calculated binding energy is approximately $-0.54 \mathrm{eV}$. The $\mathrm{E}_{\mathrm{b}}$, the minimum distances (d), $\mathrm{E}_{\mathrm{g}}$, and $\mathrm{CT}$ for optimized (8.0) SWCNT plus estrone molecule were calculated and summarized in Table 2 to the configurations shown in Figure 4. The negative values for $E_{b}$ indicate an attraction force between the molecule estrone and (8.0) SWCNT. Due to a small binding energy, small charge transfer and large binding distance we inferred that the estrone molecule was physisorbed onto the SWCNT wall.

The computed band structure of pristine SWCNT is shown in Figure 5P, to comparison. The pristine (8.0) SWCNT has the energy gap of $0.60 \mathrm{eV}$. Figures $5 \mathrm{a}-5 \mathrm{~d}$ show the computed band structure of SWCNT interacting 


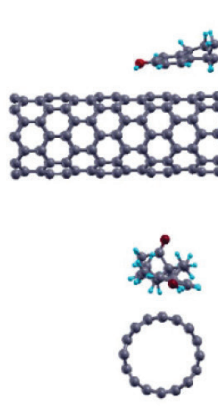

(a)
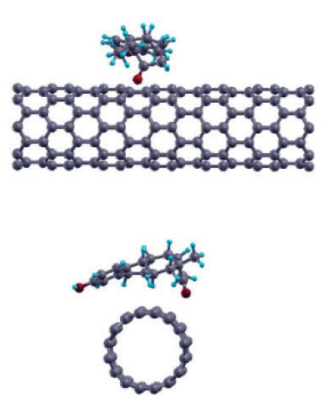

(b)
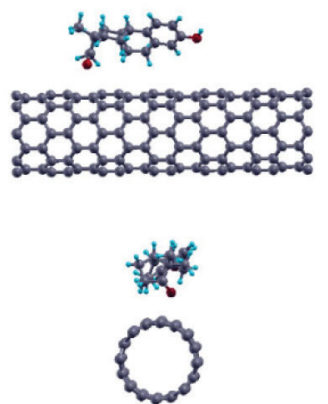

(c)
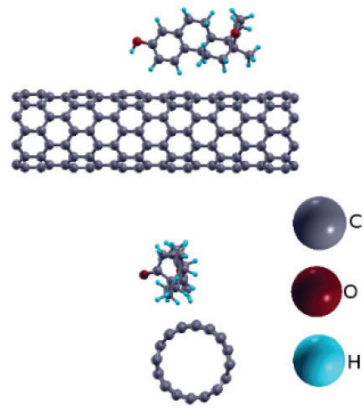

(d)

Figure 4. Top and side views of the most stable configurations for the estrone interacting with (8.0) SWCNT.

Table 2. Binding energies $\left(\mathrm{E}_{\mathrm{b}}\right)$, distance of binding $(\mathrm{d})$, gap energy $\left(\mathrm{E}_{\mathrm{g}}\right)$ and charge transfer (CT) calculated for different adsorption sites of (8.0) SWCNT, as shown in Figure 4. The minus sign in the charge transfer values indicates that estrone molecule receives electronic charge of SWCNT

\begin{tabular}{lcccc}
\hline System & $\mathrm{E}_{\mathrm{b}} / \mathrm{eV}$ & $\mathrm{d} / \AA$ & $\mathrm{E}_{\mathrm{g}} / \mathrm{eV}$ & $\mathrm{CT} / \mathrm{e}^{-}$ \\
\hline $\mathrm{a}$ & -0.54 & 2.49 & 0.58 & +0.159 \\
$\mathrm{~b}$ & -0.44 & 2.48 & 0.58 & +0.008 \\
$\mathrm{c}$ & -0.38 & 2.80 & 0.59 & -0.004 \\
$\mathrm{~d}$ & -0.32 & 2.85 & 0.59 & -0.003 \\
\hline
\end{tabular}

with estrone molecule corresponding to configurations shown in Figures $4 a-4 d$, respectively. It can be seen from the figure that after the adsorption of estrone molecule on (8.0) SWCNT, no significant electronic change has occurred. We observed that a degenerated level emerges in top of valence band in all systems and localized levels appear below the Fermi level. The electronic properties of SWCNT are unchanged by adsorption of the molecule, that is, the systems remained semiconductor with the energy gap around $0.58 \mathrm{eV}$ (see Table 2), showing the weak interaction between them, in agreement with low value of the $\mathrm{E}_{\mathrm{b}}$ and $\mathrm{CT}$.

In Figures $6 a-6 d$ the plots for the LDOS of estrone molecule adsorbed on the (8.0) SWCNT for all configurations (shown in Figure 4) are presented for the electronic levels around the Fermi level (see Figures 5a-5d). The Figure 6a, that corresponds to the most stable configuration (see Figure 4a), shows that exists contribution of nanotube atoms and the molecule atoms (mainly oxygen atoms of the molecule) in $-0.38 \mathrm{eV}$ to $0.29 \mathrm{eV}$ region (see Figure $5 \mathrm{a}$ ). In Figures $6 \mathrm{~b}$ and $6 \mathrm{c}$, the contribution to electronic properties are mainly of SWCNT and in Figure 6d the contribution to electronic properties around Fermi level are of aromatic ring of estrone molecule.

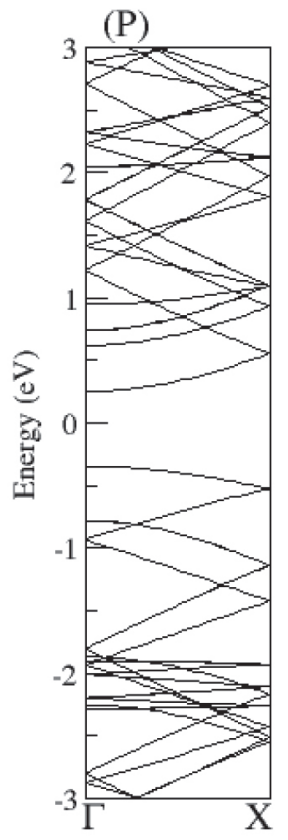

(a)

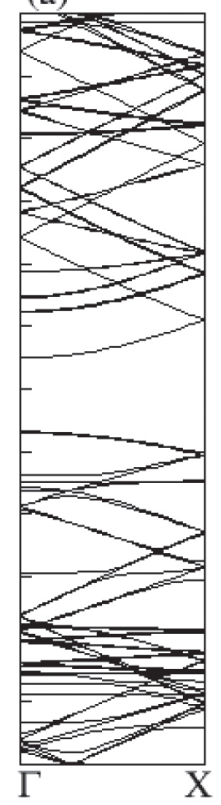

(b)

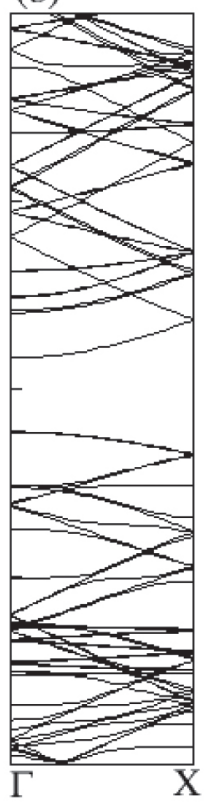

(c)

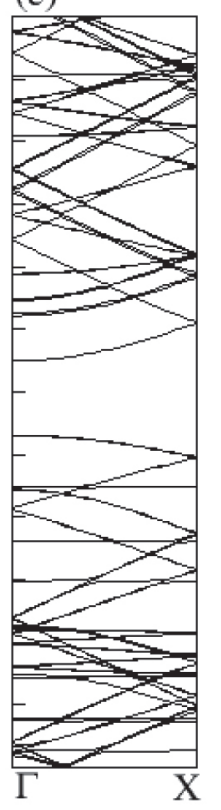

(d)

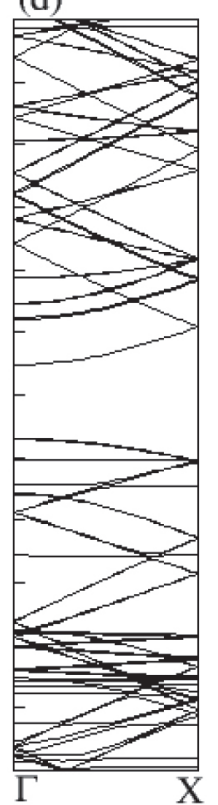

Figure 5. Calculated electronic band structure for $(\mathrm{P})(8.0)$ pristine and for (a-d) different configurations of estrone adsorbed on (8.0) SWCNT, which correspond to the configurations shown in the Figures $4 \mathrm{a}-4 \mathrm{~d}$, respectively. The Fermi energy is localized in zero. 

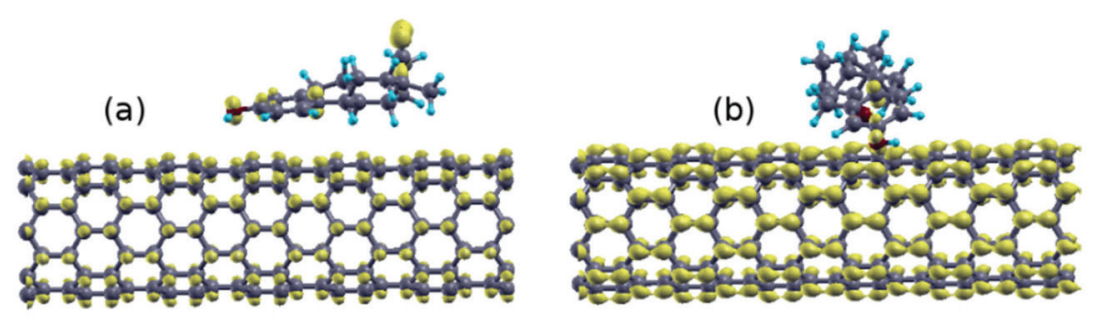

(c)
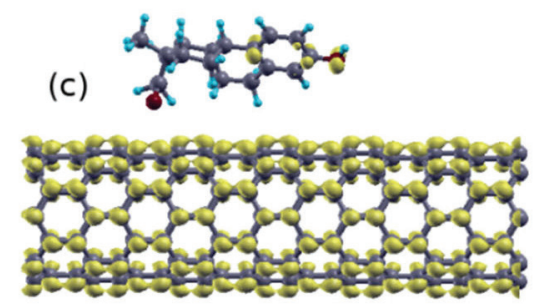

(d)
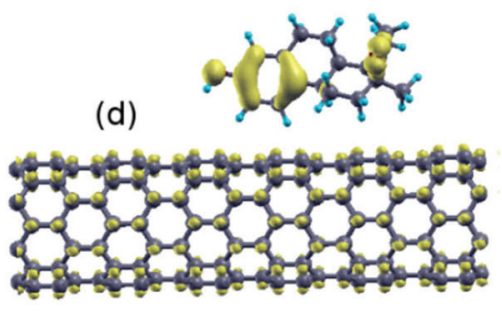

Figure 6. (a-d) Plot of the local density of states, around the Fermi level, for (8.0) SWCNT interacting with estrone molecule for the electronic configurations shown in Figures 5a-5d, respectively. An isosurface $0.003 \mathrm{e}^{-1}$ bohr $^{-3}$ was considered.

\section{Conclusions}

The $a b$ initio calculations presented in this work predicted that the interaction of ethinylestradiol and estrone molecule on the surface of (8.0) SWCNT, in different configurations, presents mostly weak binding energy, typical of physical process. The most stable configurations are those ones where the molecules are parallel to axis of the SWCNT. In particular, the ethinylestradiol molecule exhibits a tendency to receive charge of the SWCNT while the estrone molecule tends to receive or donate charge depending on its position in relation to the SWCNT. Through our results we infer that the SWCNT may be utilized as an efficient adsorbent to remove ethinylestradiol and estrone molecule from water.

\section{Acknowledgments}

We thank CENAPAD-SP for computational facilities and Fundação de Amparo à Pesquisa e Desenvolvimento Científico and Tecnológico do Maranhão (FAPEMA) for financial support. We would like to thank Prof R. S. G. Olea for inspiring discussions. S. M. C. and H. C. R. acknowledge CAPES and FAPEMA for the fellowship, respectively.

\section{References}

1. Schumock, G. T.; Li, E. C.; Suda, K. J.; Matusiak, L. M.; Hunkler, R. J.; Vermeulen, L. C.; Hoffman, J. M.; Am. J. HealthSyst. Pharm. 2014, 71, 482.

2. Ebele, A. J.; Abdallah, M. A.-E.; Harrad, S.; Emerging Contam. 2017, 3, 1 .

3. Davis, D. L.; Bradlow, H. L.; Sci. Am. 1995, 273, 166.
4. Länge, R.; Hutchinson, T. H.; Croudace, C. P.; Siegmund, F.; Schweinfurth, H.; Hampe, P.; Panteranf, C. H.; Sumpter, J. P.; Environ. Toxicol. Chem. 2001, 20, 1216.

5. Roy, D.; Colerangle, J. B.; Singh, K. P.; Front. Biosci., Landmark Ed. 1998, 3, 913.

6. Aschengrau, A.; Coogan, P. F.; Quinn, M.; Cashins, L.; Am. J. Ind. Med. 1998, 34, 6.

7. Desbrow, C. E. J. R.; Routledge, E. J.; Brighty, G. C.; Sumpter, J. P.; Waldock, M.; Environ. Sci. Technol. 1998, 32, 1549.

8. Evgenidou, E. N.; Konstantinou, I. K.; Lambropoulou, D. A.; Sci. Total Environ. 2015, 505, 905.

9. Ubertini, F.; Materazzi, A. L.; D’Alessandro, A.; Laflamme, S.; Eng. Struct. 2014, 60, 265.

10. Srivastava, A.; Srivastava, O. N.; Talapatra, S.; Ajtai, R. V.; Ajayan, P. M.; Nat. Mater. 2004, 3, 610.

11. Wang, J.; Wang, S.; J. Environ. Manage. 2016, 182, 620.

12. Li, P.; Wang, C.; Zhang, Y.; Wei, F.; Small 2014, 10, 4543.

13. Karuwan, C.; Wisitsoraat, A.; Maturos, T.; Phokharatkul, D.; Sappat, A.; Jaruwongrungsee, A.; Lomas, T.; Tuantranont, A.; Talanta 2009, 79, 995.

14. Liu, Z.; Chen, K.; Davis, C.; Sherlock, S.; Cao, Q.; Chen, X.; Dai, H.; Cancer Res. 2008, 68, 6652.

15. Kim, J. P.; Lee, B. Y.; Lee, J.; Hong, S.; Sim, S. J.; Biosens. Bioelectron. 2009, 24, 3372.

16. Das, R.; Ali, M. E.; Hamid, S. B. A.; Ramakrishna, S.; Chowdhury, Z. Z.; Desalination 2014, 336, 97.

17. Teixeira, A. P. C.; Purceno, A. D.; Paula, C. C. A.; Silva, J. C. C.; Ardisson, J. D.; Lago, R. M.; J. Hazard. Mater. 2013, 248-249, 295.

18. Machado, F. M.; Carmalin, S. A.; Lima, E. C.; Dias, S. L. P.; Prola, L. D. T.; Saucier, C.; Jauris, I. M.; Zanella, I.; Fagan, S. B.; J. Phys. Chem. C 2016, 120, 18296.

19. Machado, F. M.; Bergmann, C. P.; Lima, E. C.; Royer, B.; Souza, F. E.; Jauris, I. M.; Calvete, T.; Fagan, S. F.; Phys. Chem. Chem. Phys. 2012, 14, 11139. 
20. Jing, N.; Xue, Q.; Ji, J.; Wang, S.; Ling, C.; Comput. Mater. Sci. 2011, 50, 2909.

21. Castro, S. M.; Araújo, A. B.; Nogueira, R. F. P.; Guerini, S.; Appl. Surf. Sci. 2017, 403, 519.

22. Hohenberg, P.; Kohn, W.; Phys. Rev. 1964, 136, B864.

23. Soler, J. S.; Artacho, E.; Gale, J. D.; García, A.; Junquera, J.; Ordejón, P.; Sánchez-Portal, D.; J. Phys.: Condens. Matter 2012, 14, 2745.

24. Kohn, W.; Sham, L. J.; Phys. Rev. 1965, 140, A1133.
25. Perdew, J. P.; Zunger, A.; Phys. Rev. B 1981, 23, 5048.

26. Troullier, N.; Martins, J. L.; Phys. Rev. B 1991, 43, 1993.

27. Kleinman, L.; Bylander, D. M.; Phys. Rev. Lett. 1982, 48, 1425.

28. Monkhorstand, H. J.; Pack, J. D.; Phys. Rev. B 1976, 13, 5188.

29. Boys, S. F.; Bernardi, F.; Mol. Phys. 1970, 19, 553.

Submitted: June 21, 2019

Published online: October 10, 2019 